\title{
Control and performance improvements of a pulse compressor in use for testing accelerating structures at high power
}

\author{
Benjamin Woolley ${ }^{*}$ and Igor Syratchev \\ CERN, European Organization for Nuclear Research, 1211 Geneva, Switzerland
}

Amos Dexter

Cockcroft Institute, Lancaster University, Lancaster LA1 4YW, United Kingdom

(Received 27 March 2017; published 5 October 2017)

\begin{abstract}
New developments relating to compact X-band, SLED-I type pulse compressors being developed at CERN for testing high gradient structures are described. Pulse compressors of interest take rf pulses from one or more high power klystrons with duration typically $>1.5 \mu$ s and deliver up to 5 times the input power for a shorter duration $<250 \mathrm{~ns}$. Time domain models for pulse compressor operation with low level $\mathrm{rf}$ (LLRF) control have been developed. Input drive amplitude and phase for each pulse is evolved with a control algorithm from the pulse compressor output for previous pulses. The goal is to deliver precise amplitude for pulses to test stands and precise amplitude and phase for pulses to accelerator systems. Control algorithms have been developed and validated experimentally.
\end{abstract}

DOI: 10.1103/PhysRevAccelBeams.20.101001

\section{INTRODUCTION}

Linear accelerators (LINAC) provide multi-GeV scale charged particles for three purposes: (i) high energy physics experiments; (ii) impacting targets to produce $\mathrm{x}$ rays, neutrons or other elementary particles; and (iii) to feed free electron lasers (FELs) or undulators with electrons.

The LINAC community has a requirement to test accelerating structures at very high gradients requiring short high power rf pulses [1]. LINACS for protons typically operate at $\mathrm{rf}$ frequencies up to $1.3 \mathrm{GHz}$. LINACS for electrons typically operate at $\mathrm{rf}$ frequencies between 1.3 and $12 \mathrm{GHz}$. The acceleration gradient of a LINAC is the amount of voltage it can add to a charged particle per meter. Increasing gradient reduces length and this may reduce cost. Studies suggest that capital cost for high energy electron LINACS is minimized for $\mathrm{rf}$ frequencies near $12 \mathrm{GHz}$ [2].

CERN is currently developing test stations for conditioning and breakdown rate testing of high gradient $12 \mathrm{GHz}$ accelerating structures [3]. A goal for the development of these test stations is to reduce their cost whilst improving the peak amplitude flatness of the compressed pulse and amplitude repeatability. The LINAC accelerating structures of primary interest to CERN require testing at power levels up to $120 \mathrm{MW}$. The maximum pulse length required is $250 \mathrm{~ns}$. Whilst pulses with this duration and power level can

\footnotetext{
*benjamin.woolley@cern.ch

Published by the American Physical Society under the terms of the Creative Commons Attribution 4.0 International license. Further distribution of this work must maintain attribution to the author(s) and the published article's title, journal citation, and DOI.
}

be produced by deaccelerating high current bunched electron beams, this solution is not affordable or practical for test stations [4]. The only electronic device that can generate appropriately controlled if power at near the required power level is the klystron. State of the art X-band klystrons such as the XL5 are only able to produce $50 \mathrm{MW}$ power and have much better cathode lifetime when the power level is reduced by $30 \%$ or more [5]. These klystrons are able to produce $1.5 \mu \mathrm{s}$ pulses at their maximum power level. Short pulses with lengths between 30 and 250 ns at power levels above $100 \mathrm{MW}$ can be produced by compressing longer pulses. An established technique for compressing rf pulses is to feed $\mathrm{rf}$ energy into pairs of cavities or transmission lines with a $3 \mathrm{~dB}$ hybrid coupler and then once a set level of energy has been stored, the phase of the feed is switched by $180^{\circ}$ so as to discharge the stored energy into the device under test in a much short period [6-8]. Since their inception a small number of high power rf pulse compressors have been developed. There is still much scope for engineering improvements to their design [9-15]. Section II of this paper describes the development of high power compact $\mathrm{X}$-band pulse compressors at CERN. Section III sets out the equations applicable to modeling compact SLED-1 type pulse compressors. Section IV derives the envelope equation for time dependent analyses. Sections V and VI consider low level rf (LLRF) control strategies for pulsed systems. The methods are applicable to SLED-1 type pulse compressors utilizing two primary cavities and possibly a few additional pairs of secondary cavities $[10,15]$. Section VII discusses tuning issues. Sections VIII and IX give implementation details and experimental results.

The accelerating structures of interest when providing accelerating gradients of $100 \mathrm{MV} / \mathrm{m}$ have surface electric 
fields in excess of $200 \mathrm{MV} / \mathrm{m}$ and electric current densities in excess of $400 \mathrm{kA} / \mathrm{m}$ [15]. In order to get a copper structure to run with these levels of surface electric field and current, the power level and pulse length must be increased gradually from significantly lower levels allowing small vacuum breakdown events to gradually remove surface defects that would give damaging arcs at higher power levels. This process is known as conditioning. The breakdown rate for a typical CLIC or NLC copper accelerating structure increases with electric field raised to a power that is usually in the range 18 to 35 [1,16-18]. In order to minimize damaging arcs during the conditioning process it is very important that at its maximum, the amplitude of the pulse does not vary with time i.e. has a "flattop" and is accurately controlled to the required fixed level.

As part of the conditioning process new algorithms are being developed by CERN to minimize conditioning time whilst maximizing final performance. These algorithms dictate the way that power is reduced and then subsequently raised after a single breakdown event or a sequence of breakdown events. Due to the very high power levels in the pulse compressor, changes of power level significantly change temperatures, as a consequence the tune and the match of the rf cavities and couplers in the pulse compressor also change. This alters the pulse height and flattop performance. This theory set out in this paper encompasses the case when cavities are slightly detuned.

Variation of the rf input phase to the pulse compressor with a particular profile can generate a flattop amplitude output for some limited period. Variation of the rf input amplitude and phase with particular profiles can hold the amplitude and phase of the rf output at set points, again for a limited period. This paper simulates two types of controller. In Sec. V, simulation gives evolution of the I and Q input components necessary to deliver constant amplitude and phase output during the high power compressed pulse. In Sec. VI, simulation gives evolution of the input phase ramp necessary to deliver constant amplitude output during the high power compressed pulse while output phase is allowed to drift. The controller uses errors on the previous pulse to determine inputs for the next pulse. Giving a test station the ability to reoptimize the $\mathrm{rf}$ input after the conditioning algorithm has made significant power variations enables the system to continue high gradient testing without the intervention of an operator.

CERN has three high power facilities for testing $12 \mathrm{GHz}$ CLIC structures known as XBOXs. XBOX-2 and -3 have identical pulse compressors whilst XBOX-1 has an earlier design. XBOX-1 and -2 are powered by SLAC XL5 klystrons delivering $50 \mathrm{MW}$ for $1.5 \mu \mathrm{s}$. XBOX-3 combines power from multiple Toshiba E37113 klystrons delivering $6 \mathrm{MW}$ for $5 \mu \mathrm{s}$. Section IX gives results for the performance of the XBOX-3 pulse compressor before and after the implementation of a controller.

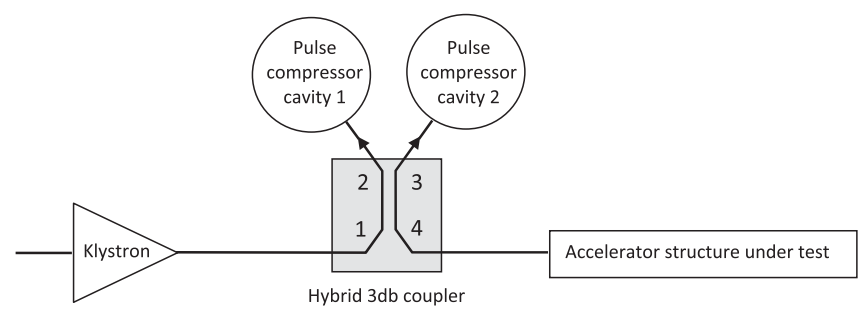

FIG. 1. Pulse compressor layout.

\section{COMPACT SLED-1 PULSE COMPRESSOR}

A SLED-I type rf pulse compressor uses two storage cavities and a hybrid $3 \mathrm{~dB}$ coupler as shown in Fig. 1. The function of the $3 \mathrm{~dB}$ hybrid coupler in a pulse compressor is to separate the wave from the klystron at port 1 that fills the pulse compressor cavities from the wave that leaves these cavities at port 4 . This is possible because the cavities are filled with a relative $90^{\circ}$ phase shift. A pulse compressor might also be formed with a circulator and a single cavity however circulators do not operate at the extremely high power levels of interest.

For the $12 \mathrm{GHz} \mathrm{XBOX}$ installations at CERN, the intrinsic quality factor $Q_{o}$ is made as high as possible by filling the cavity with a cylindrical TE01 mode. An intrinsic $\mathrm{Q}$ value of $1.80 \times 10^{5}$ was achieved for XBOX-2 and -3 . The external quality factor $Q_{e}$ is chosen so that the cavity can be filled to its maximum stored energy within the rf pulse time that the klystron can deliver. The $50 \mathrm{MW}$ klystron used at XBOX-2 delivers a $1.5 \mu$ s pulse, $Q_{e}$ was set at 30,000.

Figure 2 shows the XBOX-2 pulse compressor being tested at low power. At XBOX-3 the klystrons deliver longer pulses and $Q_{e}$ was set at 40,000.

The feature that allows the new SLED to be compact is the design of the storage cavities using adiabatic tapers at each end ensuring that unwanted modes are not produced [19]. This allows a pure TE01-like mode to exist in the cavity and simplifies the task of the mode converters. Additionally the mode converters have a very compact design.

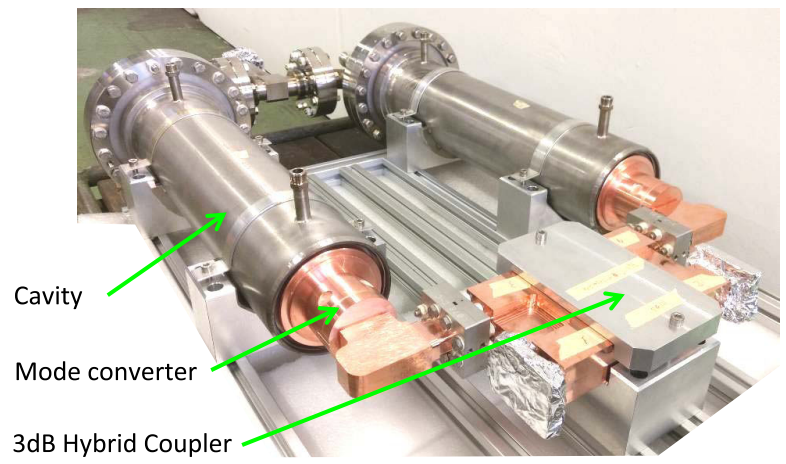

FIG. 2. SLED-I pulse compressor. 


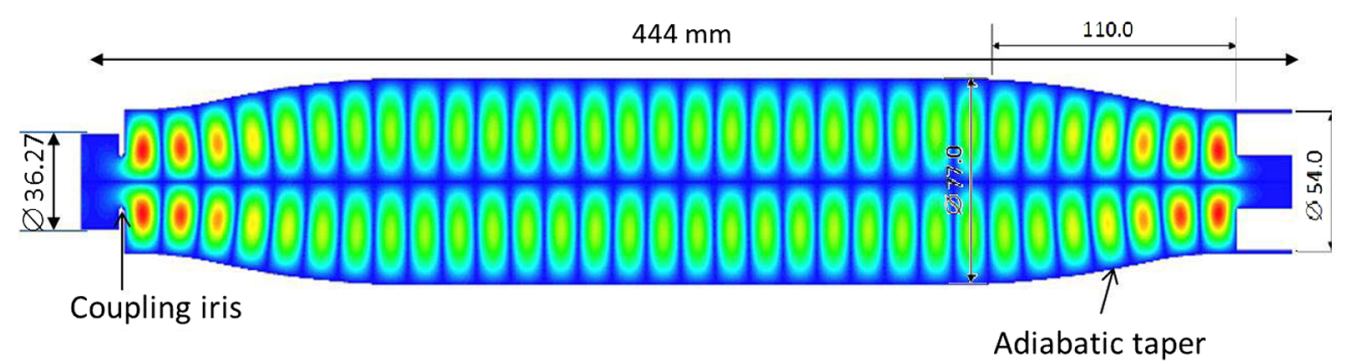

FIG. 3. Electric field pattern inside of the storage cavity of the new SLED-I [20].

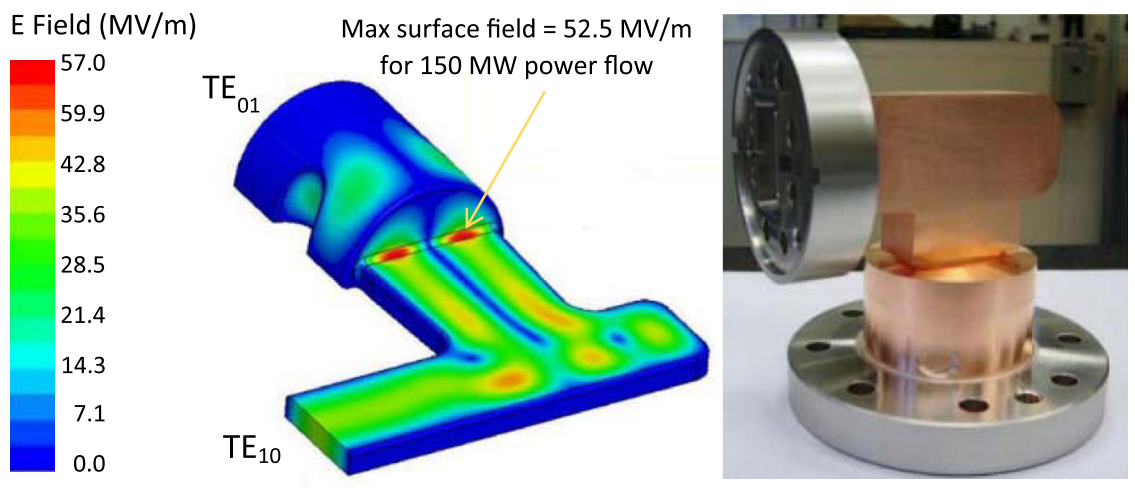

FIG. 4. (Left) Inner geometry and electric field of the mode launcher. (Right) Photograph of the compact mode converter.

Figure 3 shows the adiabatic tapers at each end of the cavity that reduce coupling of the TE02 mode to $-60 \mathrm{~dB}$ at the operating frequency. The TE03 mode is below the cutoff frequency.

Figure 4 shows the compact couplers to the pulse compressor cavities. The TE10 mode is first transformed into the TE20 mode using a shorted piece of waveguide and a right angle bend. The TE20 mode is passed into the circular waveguide with vertical posts for matching purposes. The method was devised at KEK [21] and SLAC [22] and adapted for XBOX pulse compressors at CEA Saclay.

\section{THEORY OF OPERATION}

The theory of pulse compression can be determined by the consideration of forward and emitted waves from a single cavity. Each pulse compressor cavity is modeled as an inductor, capacitor and resistor (LCR) parallel circuit. The real, time dependent, cavity voltage is given by taking the real part of the complex time dependent voltage $V$ which is determined by

$\frac{1}{L} \int V d t+C \frac{d V}{d t}+\left(\frac{1}{R_{s}}+\frac{1}{Z_{w g}}\right) V=\frac{2 F}{Z_{w g}} \exp (j \omega t)$,

where $Z_{w g}$ is the impedance of the transmission line into the cavity, $R_{s}$ is the cavity shunt impedance and $F$ is the phasor for the forward wave into the pulse compressor cavity. The phasor $F$ is given a time dependence to modify the output as required by the application. The phasor of the outgoing wave $R$ at the port of each pulse compressor cavity is determined from

$$
V(t)=\{F(t)+R(t)\} \exp (j \omega t) .
$$

The outgoing wave is the sum of the reflected and the emitted waves.

Using the definitions $\omega_{o}=1 / \sqrt{L C}, Q_{e}=\omega_{o} Z_{w g} C$, $Q_{o}=\omega_{o} R_{s} C, 1 / Q_{L}=1 / Q_{o}+1 / Q_{e}$ then (1) can be written in the familiar form (3) used for analyzing the response of a cavity:

$\frac{d^{2} V}{d t^{2}}+\frac{\omega_{o}}{Q_{L}} \frac{d V}{d t}+\omega_{o}^{2} V=\frac{2 \omega_{o}}{Q_{e}} \frac{d}{d t}\{F(t) \exp (j \omega t)\}$.

For modeling of the real system, separate equations are written for each cavity and then inputs and outputs are combined with the $\mathrm{S}$ matrix for the hybrid $3 \mathrm{~dB}$ coupler. For identical cavities and a perfect coupler, the output at port 4 can be discussed by consideration of a single cavity where ingoing and outgoing waves can be separated.

Form (1) is useful as it relates to the analysis given by [23] where a differential equation is determined whose solution gives a phase variation with time for a drive phasor $F$ of constant amplitude that generates a flattop amplitude output for a perfectly tuned pulse compressor. 
TABLE I. $\left|R\left(F_{2}\right)\right|$ after a change in the drive phasor from 1 to $F_{2}$ for $Q_{L} / Q_{e} \sim 0.85$.

\begin{tabular}{lccccc}
\hline \hline$\left|R\left(F_{2}\right)\right|$ & $\angle F_{2}=\pi$ & $\angle F_{2}=2 \pi / 3$ & $\angle F_{2}=\pi / 2$ & $\angle F_{2}=\pi / 3$ & $\angle F_{2}=0$ \\
\hline$\left|F_{2}\right|=1$ & 2.700 & 2.364 & 1.972 & 1.480 & 0.700 \\
$\left|F_{2}\right|=0.5$ & 2.200 & 2.044 & 1.841 & 1.574 & 1.200 \\
$\left|F_{2}\right|=0$ & 1.700 & 1.700 & 1.700 & 1.700 & 1.700 \\
\hline \hline
\end{tabular}

A key output from this paper is the real time determination of functions $F$ that generate flattop amplitude output when the pulse compressor is not perfectly tuned and components contribute to reflections. This section covers aspects of the underlying theory.

An exact solution for the cavity voltage phasor is easily determined from (3) when the ingoing phasor $F$ is time independent. Equation (2) then determines the time independent outgoing phasor $R$ as

$$
R=\left\{2 F \frac{Q_{L}}{Q_{e}} K \exp (j \varphi)-F\right\}
$$

where

$$
K=\omega \omega_{o} / \sqrt{Q_{L}^{2}\left(\omega_{o}^{2}-\omega^{2}\right)^{2}+\omega^{2} \omega_{o}^{2}}
$$

and

$$
\tan (\varphi)=Q_{L}\left(\omega_{o}^{2}-\omega^{2}\right) /\left(\omega \omega_{o}\right)
$$

The outgoing wave $R$ can be considered as the output at port 4 for an input at port 1 in Fig. 1.

An exact solution giving the time dependent outgoing wave $R(t)$ is also possible after a step change in the forward wave phasor from a steady value of $F_{1}$ to a steady value of $F_{2}$. It is convenient to write a more compact solution by neglecting terms that are a factor $1 / Q_{L}$ smaller than others. Assuming the phasor changes at $t=0$ and that the cavity has been filled before $t=0$ then this compact solution for the outgoing phasor is

$$
\begin{aligned}
R(t) \cong & 2 \frac{Q_{L}}{Q_{e}}\left(F_{1}-F_{2}\right) K \exp \left(-\frac{\omega_{o} t}{2 Q_{L}}+j \varphi\right) \\
& \times \exp \left\{j\left(\omega_{o}-\omega\right) t\right\}+\left\{2 F_{2} \frac{Q_{L}}{Q_{e}} K \exp (j \varphi)-F_{2}\right\} .
\end{aligned}
$$

If $\omega=\omega_{o}$ giving $K=1$ and $\phi=0$ then at $t=0$ when the drive phasor changes from $F_{1}$ to $F_{2}$ one has

$$
R(t \sim+0) \cong\left(2 F_{1} \frac{Q_{L}}{Q_{e}}-F_{2}\right) \quad \text { for } \omega=\omega_{o}
$$

For the XBOX pulse compressors under consideration $Q_{L} / Q_{e} \sim 0.83 \rightarrow 0.87$. After the drive phasor is switched the output $R$ determined by (7) decays exponentially from the values given by (8) back to its steady state level. The loaded $Q_{L}$ is made large to increase stored energy and to limit the decay time of the pulse. The external $Q_{e}$ is made large so that the instantaneous power delivered after the phasor is changed is large. Table I computes instantaneous amplitudes of the outgoing wave $R$ using (8) at the instant that the phasor changes from $F_{1}=1$ to selected new phasors $F_{2}$. Note that for $Q_{L} / Q_{e} \sim 0.85$ the steady state output $R$ is 0.7 of the input.

When the initial phase change of the ingoing phasor is less than $\pi$ its phase can be further increased with time and indeed increased in such a way that the amplitude of the outgoing amplitude stays constant. If the amplitude of the ingoing phasor is reduced initially then the outgoing amplitude can also be maintained by increasing the ingoing amplitude. If only the phase of the input is varied then whilst constant amplitude can be maintained on the outgoing pulse, the phase of the output will vary. If constant phase and amplitude is required simultaneously on the output then both phase and amplitude must be varied on the input. Higher power gain can be achieved for a fixed length pulse of constant amplitude when only the phase is varied.

\section{ENVELOPE EQUATIONS FOR TIME DEPENDENT ANALYSIS}

For modeling and analysis it is convenient to have a differential equation for slowly varying phasors rather than using (1). In Eqs. (4) and (7) the outgoing wave is recovered as $\operatorname{Re}\{R \exp (j \omega t)\}$, where $\omega$ is the angular frequency of the ingoing wave. In a real situation the resonant frequency of the cavity will have an offset to the frequency of the ingoing wave. When the phase of the ingoing wave is permitted to change then we choose its frequency so that the phase stays between $-\pi$ and $\pi$. To obtain a simplified equation for the phasors we consider deviations of the cavity voltage from the resonant frequency $\omega_{o}$ rather than the Klystron frequency $\omega$. Assume a solution of (3) of the form

$$
V=V_{c}(t) \exp \left(j \omega_{o} t\right)
$$

hence $V_{c}$ satisfies

$$
\begin{aligned}
\ddot{V}_{c} & +\omega_{o}\left(2 j+\frac{1}{Q_{L}}\right) \dot{V}_{c}+j \frac{\omega_{o}^{2}}{Q_{L}} V_{c} \\
& =\frac{2 \omega_{o}}{Q_{e}}(\dot{F}+j \omega F) \exp \left\{j\left(\omega-\omega_{o}\right) t\right\} .
\end{aligned}
$$


This manipulation is useful when $V_{c}(t)$ is slowly varying in time.

If ingoing wave phasor $F$ is slowly varying then the cavity phasor $V_{c}$ will also be slowly varying hence in (10) one can ignore its second derivative. As $Q_{L}$ is of the order of 30000 then its reciprocal is negligible compared to $2 j$. If $F$ is slowly varying with respect to the frequency $\omega$ then $|\dot{F}| \ll|\omega F|$. With these three approximations (10) becomes

$$
\frac{2 Q_{L}}{\omega_{o}} \dot{V}_{c}+V_{c}=\frac{2 \omega Q_{L}}{\omega_{o} Q_{e}} F \exp \left\{j\left(\omega-\omega_{o}\right) t\right\}
$$

which can also be written

$$
\begin{aligned}
& \frac{2 Q_{L}}{\omega_{o}} \dot{V}_{c} \exp \left\{j\left(\omega_{o}-\omega\right) t\right\}+V_{c} \exp \left\{j\left(\omega_{o}-\omega\right) t\right\} \\
& \quad=\frac{2 \omega Q_{L}}{\omega_{o} Q_{e}} F .
\end{aligned}
$$

In this expression $V_{c}$ is the phasor for $\exp \left(j \omega_{o} t\right)$ and $F$ is the phasor for the signal $\exp (j \omega t)$.

Using (2) and (9) the outgoing phasor referenced to $\omega$ is now given as

$$
R=-F+V_{c} \exp \left\{j\left(\omega_{o}-\omega\right) t\right\} .
$$

Eliminating $V_{c}$ from (12) using (13) gives

$$
\begin{aligned}
\dot{R} & +\left\{\frac{\omega_{o}}{2 Q_{L}}-j\left(\omega_{o}-\omega\right)\right\} R \\
& =-\dot{F}+\left\{\frac{\omega}{Q_{e}}-\frac{\omega_{o}}{2 Q_{L}}+j\left(\omega_{o}-\omega\right)\right\} F .
\end{aligned}
$$

This is equivalent to Eq. (8) in [23] with the addition of a complex term to address the case when the pulse compressor cavity is not perfectly tuned. Previous analyses $[24,25]$ of the CTF3 pulse compressors and the FERMI linac also include the case when the pulse compressor cavities are not perfectly tuned.

For numerical solution it is convenient to decompose (13) into its real and imaginary components with the substitutions $R=R_{x}+j R_{y}$ and $F=F_{x}+j F_{y}$ to give

$$
\begin{aligned}
\dot{R}_{x} & +\frac{\omega_{o}}{2 Q_{L}} R_{x}-\left(\omega-\omega_{o}\right) R_{y} \\
& =-\dot{F}_{x}+\left(\frac{\omega}{Q_{e}}-\frac{\omega_{o}}{2 Q_{L}}\right) F_{x}+\left(\omega-\omega_{o}\right) F_{y} \\
\dot{R}_{y} & +\frac{\omega_{o}}{2 Q_{L}} R_{y}+\left(\omega-\omega_{o}\right) R_{x} \\
& =-\dot{F}_{y}+\left(\frac{\omega}{Q_{e}}-\frac{\omega_{o}}{2 Q_{L}}\right) F_{y}-\left(\omega-\omega_{o}\right) F_{x} .
\end{aligned}
$$

Simulation results in the subsequent sections have assumed the layout in Fig. 1 where the output from the klystron is split with a hybrid $3 \mathrm{~dB}$ coupler so that the two pulse compressor cavities are filled with a $90^{\circ}$ phase shift. Equations (15) and (16) are then integrated forwards in time for each cavity which may have differing natural frequencies, $Q$ factors and couplings. Recombination of outputs from the two pulse compressor cavities in the hybrid coupler gives the phasor transmitted to the accelerator structure under test. The hybrid coupler shifts the contribution of each cavity by $90^{\circ}$, one cavity gets the shift on the way in and the other gets the shift on the way out. For the discussion on control (but not for the simulations) we consider the control of an individual cavity by manipulating $\mathrm{F}$ in response to an error on $R$. In practice the control must be applied to the whole compressor. Corrections to the pulse compressor input, based on error measurements on the pulse compressor output must be rotated by $90^{\circ}$ in phase space with respect to $R$ in (15) and (16).

\section{IQ CONTROL DELIVERING STEADY AMPLITUDE AND PHASE}

For short pulse applications such as CLIC where the pulse length is only $200 \mathrm{~ns}$ there is insufficient time to measure the output $\mathrm{R}$ and then make an adjustment to the input $\mathrm{F}$ to maintain a steady output. Were it possible to make feedback on the time scale of nanoseconds then Eqs. (15) and (16) nominally predict how to change $F$ when $R$ is to be maintained at its current values so that $\dot{R}=0$. For a real system the natural frequencies of the cavities will have slight variations with temperature and vibration and therefore cannot be assumed to be known precisely. This means that the solution of (14) is not a good way to determine the necessary input. Instead a controller that looks at output errors is used to determine the input. When there is no time to make corrections during a pulse the error must be recorded during a pulse and corrections made for the next pulse.

For continuous wave and long pulse applications one would measure $R_{x}$ and $R_{y}$ which are the $I$ and $Q$ components of the outgoing wave at port 4 and then adjust $F_{x}$ and $F_{y}$ with a real time controller. If the aim is to keep the $R_{x}$ and $R_{y}$ at fixed set point values $S_{x}$ and $S_{y}$ then the error terms are $\left(R_{x}-S_{x}\right)$ and $\left(R_{y}-S_{y}\right)$. The initial values of $R_{x}$ and $R_{y}$ at the start of the compressed pulse are determined by the instantaneous changes in the drive inputs $F_{x}$ and $F_{y}$. If the initial value is the desired value then for the duration of the pulse one only needs to keep $\dot{R}_{x}=0$ and $\dot{R}_{y}=0$; hence, the error can be regarded simply as the derivative of the output. For a feed forward controller we seek functions $H$ that determine inputs for the next pulse from inputs for the current pulse, i.e.

$$
F_{x}(n+1, t)=F_{x}(n, t)+H_{x}\left\{\dot{R}_{x}(n, t), \dot{R}_{y}(n, t)\right\}
$$


$F_{y}(n+1, t)=F_{y}(n, t)+H_{y}\left\{\dot{R}_{x}(n, t), \dot{R}_{y}(n, t)\right\}$,

where $F_{x}(n, t)$ and $F_{y}(n, t)$ are inputs for the $n$th pulse.

The input-output relationship for the in-phase component of the pulse compressor as given by (14) is of the form

$$
\dot{R}_{x}=-\dot{F}_{x}+G\left(R_{x}, R_{y}, F_{x}, F_{y}\right) .
$$

Phasors $F_{x}, F_{y}, R_{x}$ and $R_{y}$ must be continuous functions of time as neither the klystron nor the pulse compressor cavities can instantly step change their fields. This means that if the current value of $\dot{F}_{x}(n, t)$ in (19) results in $\dot{R}_{x} \neq 0$ then it can always be changed (within the limits of the klystron maximum output) to bring $\dot{R}_{x}=0$. Stated another way an error in the time derivative of $R$ can be instantly corrected with an opposite change in the time derivative of $F$. This means that the controller for making corrections on the next pulse is simpler than that of (17) and (18) as one only requires

$$
\begin{aligned}
& \dot{F}_{x}(n+1, t)=\dot{F}_{x}(n, t)+\dot{H}_{x}\left\{\dot{R}_{x}(n, t)\right\} \\
& \dot{F}_{y}(n+1, t)=\dot{F}_{y}(n, t)+\dot{H}_{y}\left\{\dot{R}_{y}(n, t)\right\} .
\end{aligned}
$$

Numerical simulation and experiment show that rapid convergence to flattop output for $I$ and $Q$ components is found with a proportional, integral controller applied to each component, i.e.

$\dot{F}(n+1, t)=\dot{F}(n, t)+\alpha_{p} \dot{R}(n, t)+\alpha_{i}\{R(n, t)-S\}$,

where $\alpha_{p}$ is the proportional coefficient and $\alpha_{i}$ is the integral coefficient. The coefficient $\alpha_{p}$ is designated the proportional coefficient as the correction to the time derivative of the forward wave is proportional to the time derivative of the error of the outgoing wave.

Considering now the pulse compressor with two storage cavities as shown in Fig. 1, take $F$ as the input phasor, define $T$ as the output phasor and then accounting for the $90^{\circ}$ rotation in the hybrid coupler then the controller becomes

$\dot{F}_{x}(n+1, t)=\dot{F}_{x}(n, t)-\alpha_{p} \dot{T}_{y}(n, t)+\alpha_{i}\left[T_{y}(n, t)-T_{y s p}\right]$

$\dot{F}_{y}(n+1, t)=\dot{F}_{y}(n, t)+\alpha_{p} \dot{T}_{x}(n, t)-\alpha_{i}\left[T_{x}(n, t)-T_{x s p}\right]$,

where coefficients $\alpha_{p}$ and $\alpha_{i}$ are positive, $T_{x s p}$ and $T_{y s p}$ are set points for the $I$ and $Q$ components of the output. Application of this algorithm must be delayed until the amplitude has risen in direct response to the phase switch. This rise time is determined by the bandwidth of the klystron. The set points might be taken from the maximum amplitude near the start of a pulse.

Figure 5 shows amplitudes and phases for input voltage $F$ and transmitted voltage $T$ as determined by solving envelope Eqs. (15) and (16) for each cavity for the split input, combining voltages with the hybrid coupler and then applying controllers (23) and (24). The calculation is repeated with the iterative controller until the transmitted wave no longer has an observable variation with iteration number.

The pulse compressor cavity data used corresponds to the system shown in Fig. 2, $Q_{o}=180,000, Q_{e}=40,000$. The input rf pulse from the klystron starts at $-1850 \mathrm{~ns}$ and lasts until $280 \mathrm{~ns}$ hence is on for $2130 \mathrm{~ns}$. The initial input

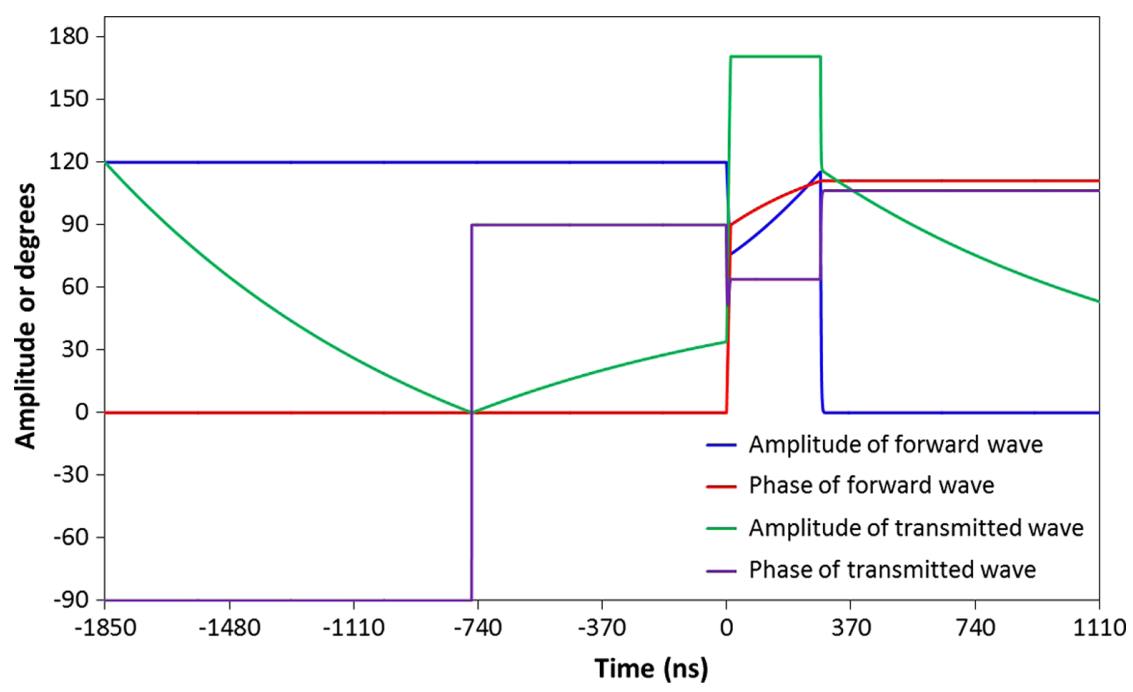

FIG. 5. Simulated pulse after application of the feed forward algorithm for several iterations until convergence is achieved. 


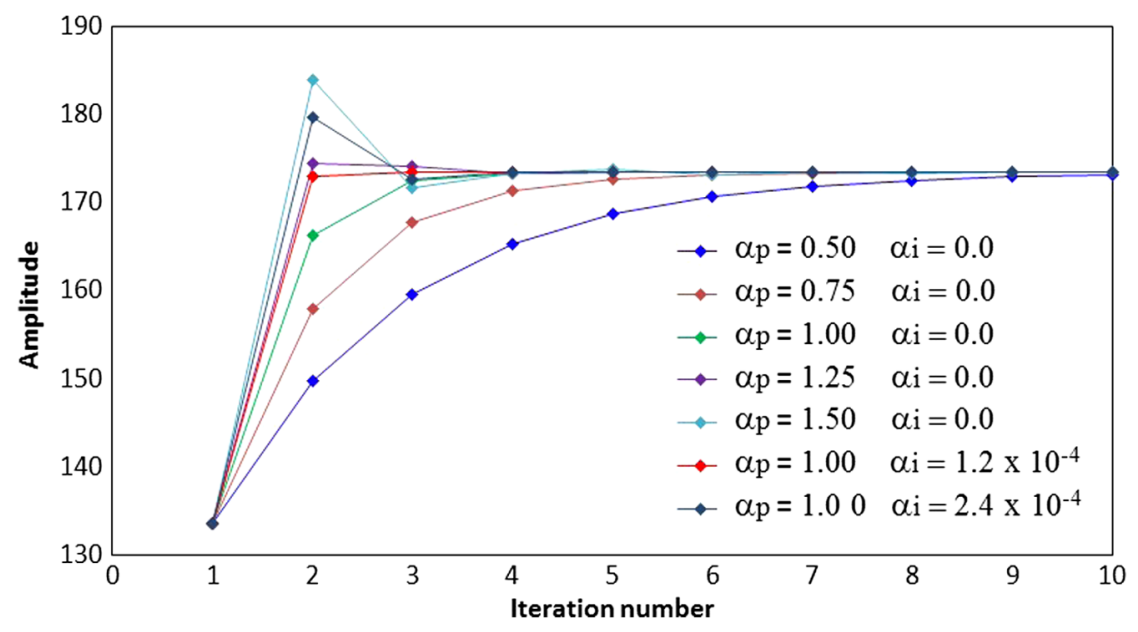

FIG. 6. Convergence of pulse amplitude as a function iteration number at time $266 \mathrm{~ns}$ after the phase flip for differing controller coefficients.

amplitude was taken as 120 so amplitudes could be plotted on the same scale as degrees. The phase flip occurring at $t=0$ is a smooth clockwise rotation of 90 degrees with a reduction in amplitude to 76 in a time permissible by a $0.3 \%$ bandwidth, i.e. $I Q$ goes from $(120,0)$ to $(0,76)$. In this example the output amplitude is shown in green and the output phase shown in purple, they are controlled perfectly. The output amplitude for the compressed pulse is 170.8 hence the power increase is a factor of 2.025. The computational time step was one rf period at $12 \mathrm{GHz}$. When the control coefficients were chosen as $\alpha_{p}=1.0$ and $\alpha_{i}=1.2 \times 10^{-4}$ the average error on the derivative of the outgoing wave was being reduced by 1 order of magnitude per iteration. The figure gives signals after the seventh iteration. The source code used for this simulation has been made open source [27].

Figure 6 shows how convergence depends on the proportional and integral coefficients. In this figure convergence is monitored by observing the amplitude at a time near the end of the pulse. For computational solutions without measurement errors, taking $\alpha_{p}=1.0$ and $\alpha_{i}=0.0$ gives excellent convergence. Increasing the proportional term slightly above 1.0 improves convergence on the second iteration but not on subsequent iterations. In an experimental situation one requires an integral term in case the set point amplitude is not achieved at an identical time on each pulse. The proportional term gives a flattop only with respect to the amplitude of a chosen measurement near the start of the pulse. A reduced proportional term will reduce the chance of over voltage in the presence of measurement errors.

Figure 7 shows the convergence sequence for the output amplitude using a suboptimal choice of control coefficients with $\alpha_{p}=0.5$ and $\alpha_{i}=0.0$. Iteration 1 in Fig. 7 shows the natural decay of the output for a steady input after the phase flip.

\section{USING A PHASE RAMP TO CONTROL ONLY THE AMPLITUDE}

For applications such as structure conditioning and testing there is no requirement to maintain a steady phase during the high power rf pulse. In this instance one can achieve higher compression with steady amplitude by varying just the phase of the input. It is also possible to achieve a steady output amplitude by just varying the input amplitude after a phase

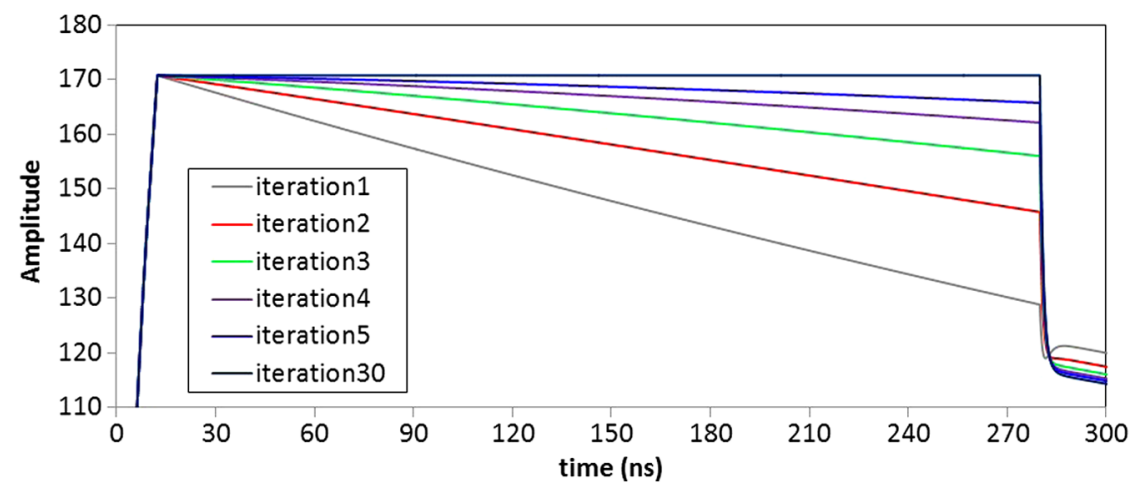

FIG. 7. Amplitude during pulse for successive iterations with control coefficients $\alpha_{p}=0.5$ and $\alpha_{i}=0.0$. 


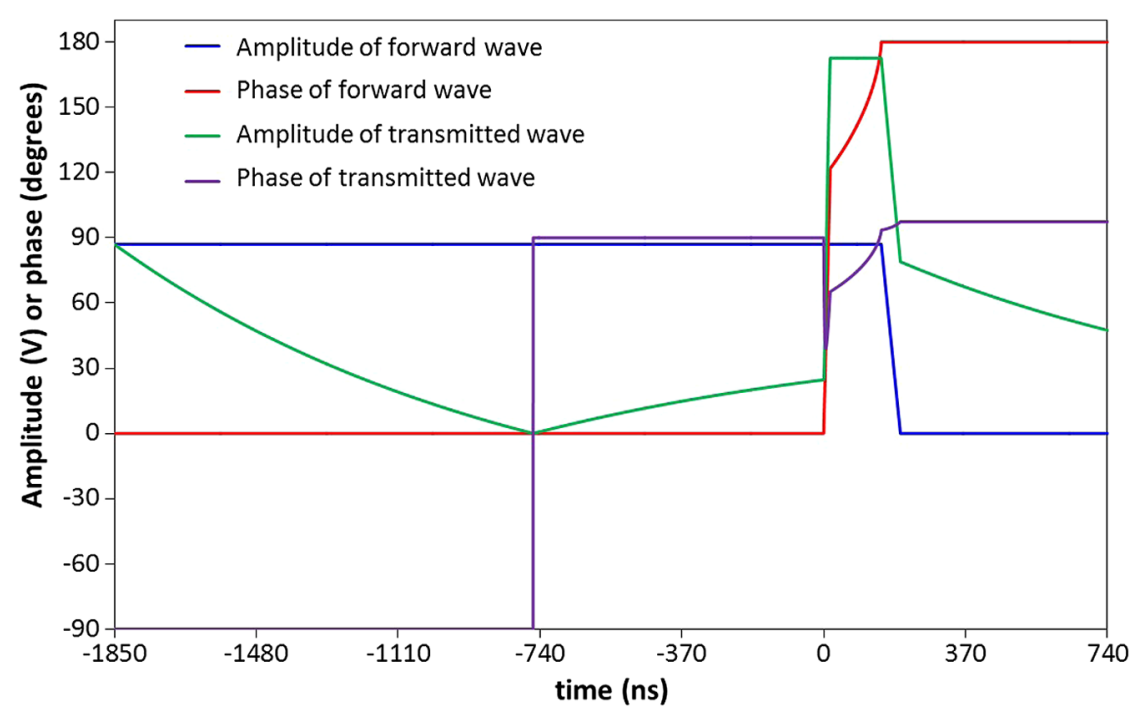

FIG. 8. Simulated performance for amplitude control using a phase ramp.

switch [26]. This possibility is not considered here as the $\mathrm{X}$-band klystrons in use have a very nonlinear response to large amplitude variations on the input.

The phase program to generate a flat amplitude for an ideal pulse compressor has been computed by [23]. Here a controller that determines the phase program for an imperfect pulse compressor whose properties may vary with time is needed. From (23) and (24) in Sec. V the errors in the $I$ and $Q$ component of the transmitted wave determine corrections to the $I$ and $Q$ components of the ingoing wave. In this section an error in the transmitted amplitude determines a correction to the phase of the ingoing wave. The amplitude of the ingoing wave is held constant and the phase of the transmitted wave varies without any control constraint.

Defining $\theta$ as the phase of the input $F$ whose magnitude is kept constant then (23) and (24) suggest a controller of the form

$\dot{\theta}(n+1, t)=\dot{\theta}(n, t)+\alpha_{p} \frac{d|T(n, t)|}{d t}-\alpha_{i}\left[|T(n, t)|-T_{s p}\right]$.
This is a nonlinear controller hence stability and convergence is more difficult to predict than for the $I Q$ controller of the last section. Figure 8 shows amplitudes and phases for input voltage $F$ and transmitted voltage $T$ as determined by solving envelope Eqs. (15) and (16) for each cavity after splitting, then combining with the hybrid coupler and applying controller (25). Results are given after the controller has converged.

At time $t=0$ the input phasor rotates with constant amplitude from $0^{\circ}$ to $122^{\circ}$. This initial phase change just permits an output pulse of length $150 \mathrm{~ns}$ which maintains constant amplitude; it can be seen that the input phase is just short of $180^{\circ}$ at the end of the pulse. Overall power gain is 3.93. Parameters for the cavities were identical to the case of Fig. 5 where control provided constant amplitude and phase on the output, but with a much reduced power gain of 2.025 and for a much longer time of $280 \mathrm{~ns}$.

Figure 9 shows power output associated with Fig. 8. This plot can be compared with experimental results to be given later. The source code used for this simulation has been made open source [27].

Figure 10 shows how the algorithm for the phase/ amplitude control converges for differing controller

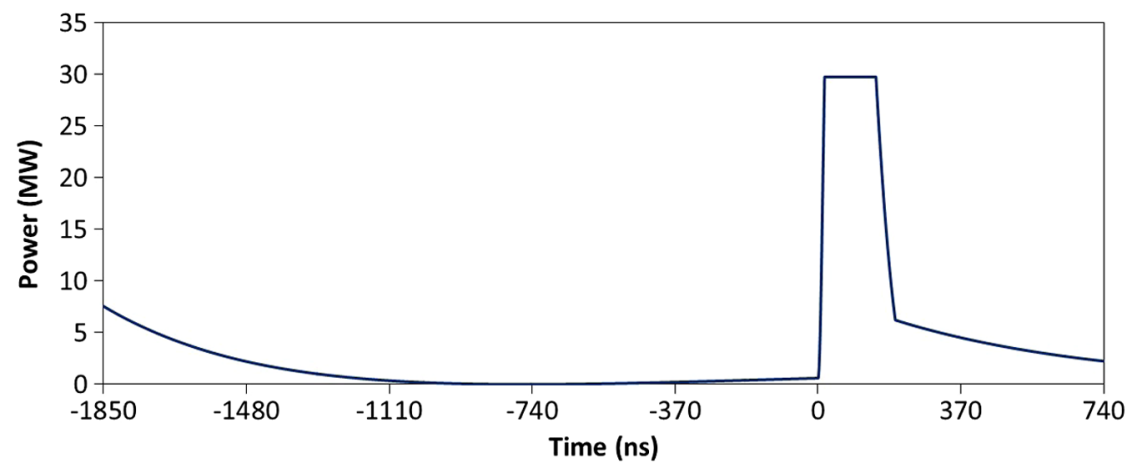

FIG. 9. Simulated power output for amplitude control using a phase ramp. 


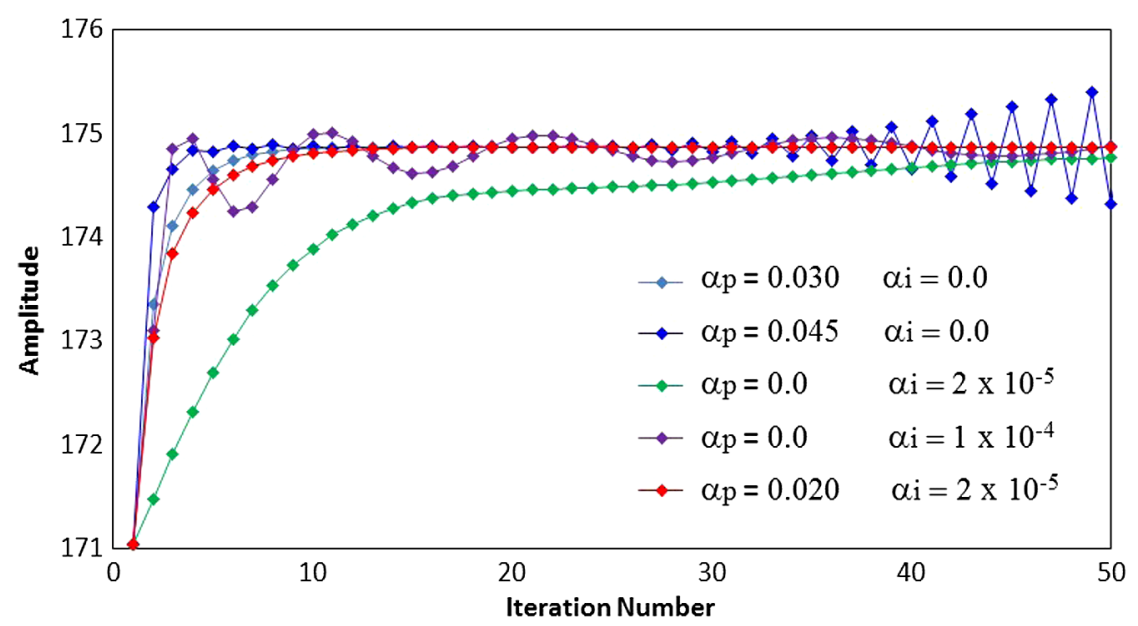

FIG. 10. Convergence of pulse amplitude as a function iteration number at time $142.5 \mathrm{~ns}$ after the start of the pulse for differing controller coefficients.

coefficients. The figure only considers the amplitude of the outgoing wave at a time near to the end of the pulse. The controller converges far more slowly than the $I Q$ controller of Sec. V. Optimal choice of $\alpha_{p}$ and $\alpha_{i}$ allowed the average error on the derivative of the outgoing wave to be reduced by about a factor of 2 per iteration. The integral controller $\left(\alpha_{p}=0\right)$ was quite poor leaving a small persistent ripple on the flattop that needed hundreds of iterations to damp away.

\section{PULSE COMPRESSOR TUNING}

At the first X-band test stand set up at CERN known as XBOX-1, the pulse compressor was an earlier design than described in Sec. II. The pulse compressor cavities had tuning plungers. A flaw in the design was that the tuners moved differentially with respect to the pulse compressor cavities as they changed temperature. The pulse compressor temperature changes after forward power is reduced. It is frequently necessary to reduce power after multiple rf breakdown events in close succession. The design flaw was eliminated for the XBOX-2 facility but to get satisfactory performance from the XBOX-1 facility an algorithm to retune the cavities was developed. The XBOX-2 and -3 pulse compressors have no tuning pistons but use a cooling system to maintain temperature at the level of $0.1^{\circ} \mathrm{C}$. Tuning is possible by changing the temperature set point.

Solving the envelope Eqs. (15) and (16) determines the effect of detuning. Figure 11 gives solutions for the computed response for nine detuning possibilities. In this figure the two pulse compressor cavities $A$ and $B$ either have their natural frequency increased by $100 \mathrm{kHz}$, left the same or decreased by $100 \mathrm{kHz}$. There are nine possibilities but three pairs are identical therefore only six responses are plotted. The phase program in each case is that which would give a flat response when cavities $A$ and $B$ are tuned exactly to the drive frequency. This case is plotted with the key $A, B$. The figure shows that a flattop compressed pulse might alternatively be achieved by changing the cavity tune

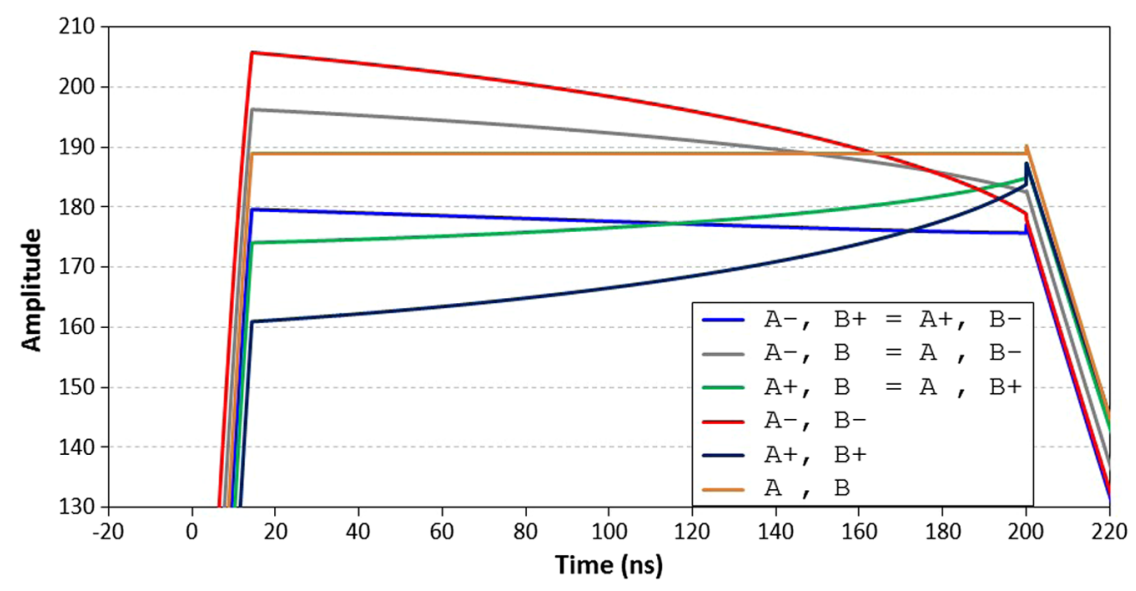

FIG. 11. Simulation of the effect of changing the natural frequency of the pulse compressor cavities. In the key, $A^{+}$and $B^{+}$means cavities $A$ and $B$ have had their frequency increased by $100 \mathrm{kHz}$ respectively, $A^{-}$and $B^{-}$means natural frequencies decreased by $100 \mathrm{kHz}$ and $A$ and $B$ means that natural frequencies stayed the same. 


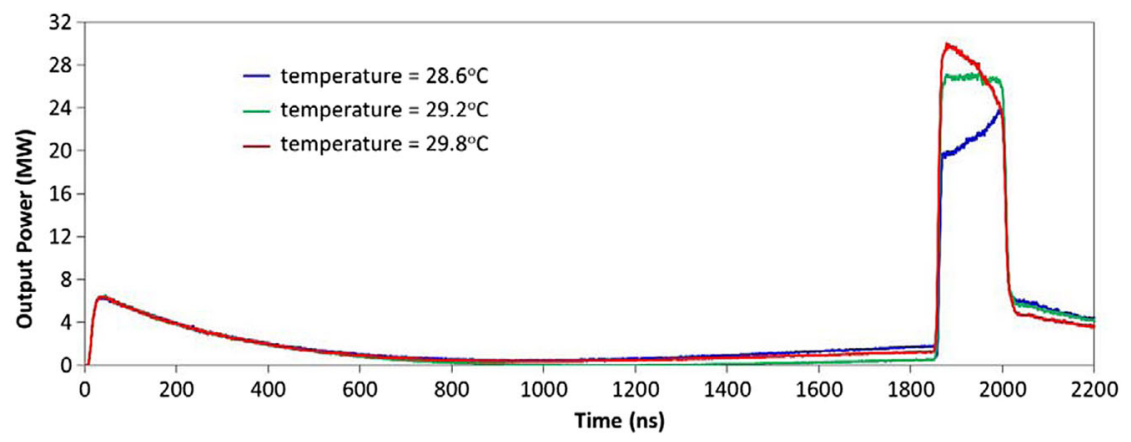

FIG. 12. Measured pulse shape at XBOX-3 when pulse compressor cavities are detuned by heating above or below the temperature from perfect tune.

rather than by applying a controller to the phase program. The figure shows that if the pulse top starts high and then reduces then the cavities need to be tuned to a higher frequency and if the pulse top starts low and then increases then the cavities need to be tuned to a lower frequency. This situation does however reverse depending on whether the phase jump at the start of the compressed pulse is positive or negative.

Figure 12 gives measured transmitted output from the XBOX-3 facility when the pulse compressor cavities are detuned by changing their temperature. Comparison with Fig. 11 shows that increasing the temperature from perfect tune reduces the frequency as expected. The tune is more easily determined by looking at either reflected power on the input to the pulse compressor or the phase of the transmitted power during filling as this does not depend on the phase program being used to get a flattop.

Figure 13 shows that if the cavity is below the optimum temperature the transmitted phase will initially decrease during filling and if above it will increase. The output pulse shape in Fig. 12 corresponds to the average frequency of the two cavities. Consideration of reflected power gives the frequency difference between the pulse compressor cavities. Energy reflected back to the klystron in the pulse up to the phase switch and beyond the phase switch is measured separately and divided to obtain a ratio. The ratio is related to the sign of the natural frequency mismatch between the two cavities. Table II gives the fuzzy logic tuning algorithm employed.

\section{IMPLEMENTATION OF MEASUREMENT AND CONTROL}

The precise CLIC frequency is $11.994 \mathrm{GHz}$ rather than $12 \mathrm{GHz}$ but frequencies quoted will be rounded to three significant figures. Measurement of the in-phase and quadrature components of the $12 \mathrm{GHz}$ rf from the pulse compressor is made after mixing with an $11.6 \mathrm{GHz} \mathrm{LO}$. After low pass filtering the down mixed signal at $400 \mathrm{MHz}$ is digitally sampled. The minimum intermediate frequency is set by time scales and frequencies of interest in the output. Sampling is performed by a National Instruments PXI system. A NI 5772 analog-to-digital converter (ADC) provides 12 bit samples at $1.6 \mathrm{GS} / \mathrm{s}$. Mixing to $400 \mathrm{MHz}$ allows quadrature sampling so that in-phase and quadrature field components and their negatives are given on four consecutive samples. The effective number of bits (ENOB) for the NI 5772 card is reduced by noise to 9.5 hence, sample to sample, $I$ and $Q$ cannot be measured to better than $0.14 \%$. Measured amplitudes determined from adjacent samples (0.625 ns spacing) for the actual system have a typical variance of $0.4 \%$. Effects that degrade performance beyond the ENOB contribution include ADC clock jitter, channel cross talk and a noisy electrical environment.

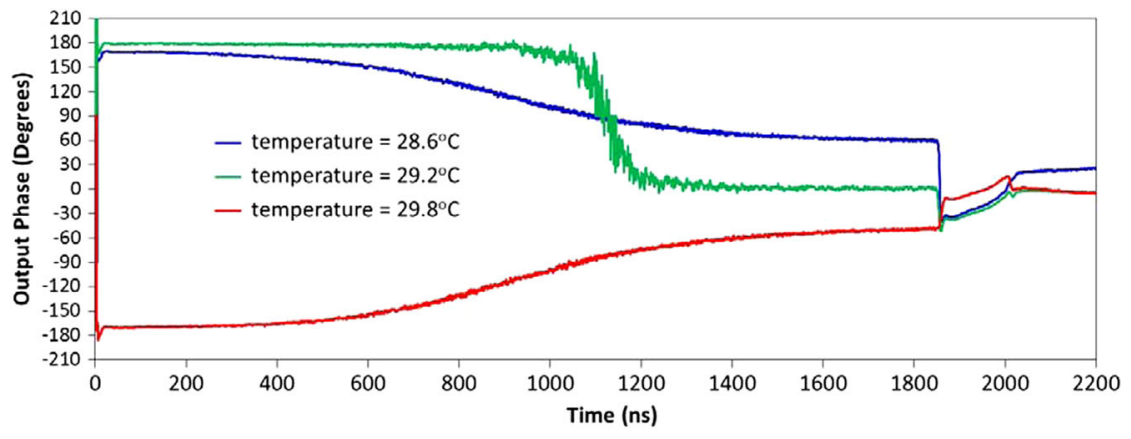

FIG. 13. Measured transmitted phase at XBOX-3 when pulse compressor cavities are detuned by heating above or below the temperature for perfect tune. 
TABLE II. Fuzzy logic tuning algorithm for pulse compressor cavities at XBOX-1.

\begin{tabular}{llll}
\hline \hline & \multicolumn{1}{c}{ High refection ratio } & \multicolumn{1}{c}{ Correct reflection ratio } & Low reflection ratio \\
\hline Fill minimum late & Reduce frequency cavity $A$ & Reduce frequency both cavities & Reduce frequency cavity $B$ \\
Fill minimum correct & Small increase frequency $B$ & Do nothing & Small increase frequency $A$ \\
Fill minimum early & Increase frequency cavity $B$ & Increase frequency both cavities & Increase frequency cavity $A$ \\
\hline \hline
\end{tabular}

The initial generation step for the input rf signal with phase and amplitude ramps is performed using a NI5611 card to vector modulate a $2.4 \mathrm{GHz}$ signal. The modulated signal is then mixed with a $9.6 \mathrm{GHz} \mathrm{LO}$ generating the modulated $12 \mathrm{GHz}$ input. $I$ and $Q$ inputs for the vector modulator are digitally generated with a 16 bit, $200 \mathrm{MS} / \mathrm{s}$, NI5450 arbitrary signal generator. The inputs to the vector modulator are low pass filtered at $100 \mathrm{MHz}$ hence rf applied to the Klystron has its bandwidth restricted to $\pm 100 \mathrm{MHz}$. The LO oscillators at 11.4 and $9.6 \mathrm{GHz}$ are derived by multiplying 2.9 and $2.4 \mathrm{GHz}$ signals by 4 . The 2.9 and $2.4 \mathrm{GHz}$ signals are locked together with a $10 \mathrm{MHz}$ reference. Full details are provided in [20].

\section{PERFORMANCE AFTER IMPLEMENTATION OF CONTROLLER}

The XBOX-1 and XBOX-2 LLRF systems were initially configured to accept predetermined phase ramps. The first automatic controller employed utilized the algorithm defined by (25) with $\alpha_{p}$ set to zero. The coefficient $\alpha_{p}$ multiplies a derivative of the output. When $\alpha_{p}$ is nonzero, noise on the amplitude measurement at the level of $0.4 \%$ in $0.625 \mathrm{~ns}$ requires smoothing before this term can enhance control. With $\alpha_{p}=0$ the controller defined by (25) was found to be unstable. On activation there can be some flattening of the high power compressed output, but after time, flattening deteriorates. For high power testing a temporary solution was to allow the algorithm to partly evolve the input phase ramp to the point of deterioration, then with the automatic control off minor adjustments made by hand. The system then operates with this fixed predetermined phase program.

The $I Q$ controller defined by (23) and (24) has not been considered for use at XBOX. This is partly because controlling the phase of the output pulse is of no interest to the high gradient testing program. There is an additional issue in that the nominal bandwidth for the klystrons only applies for small changes in the amplitude and transients after switching get significantly worse for large changes in the amplitude. One should note that the analysis of Sec. V did not include the klystron transfer function. $I Q$ control has yet to be implemented. In order to avoid disruption of the high power testing program further development of the controller has been undertaken at XBOX-3 during its commissioning phase. $\mathrm{XBOX}-3$ combines power from two smaller E37113 klystrons. Operation at $7 \mathrm{MW}$ for $2 \mu \mathrm{s}$ and with a pulse power gain factor of 5 makes very short output pulses (140 ns) with a peak power of $70 \mathrm{MW}$ achievable.

Figure 14 plots measured input amplitude and phase from a single klystron to the pulse compressor and plots output amplitude and phase from the pulse compressor into a load at XBOX-3. Smoothing of the raw sampled data has been applied prior to plotting. With respect to results in Fig. 14, the drive signal to the preamplifier has a constant amplitude, the phase switches by $+120^{\circ}$ at $1840 \mathrm{~ns}$ after which there is a perfectly linear phase ramp until $180^{\circ}$ is reached. The output from the klystron which is the input to the pulse compressor has huge transients after the phase switch. It should be noted that the XL5 klystron used at XBOX-1 and -2 has smaller transients after the phase switch. Associated with the amplitude transient is a much

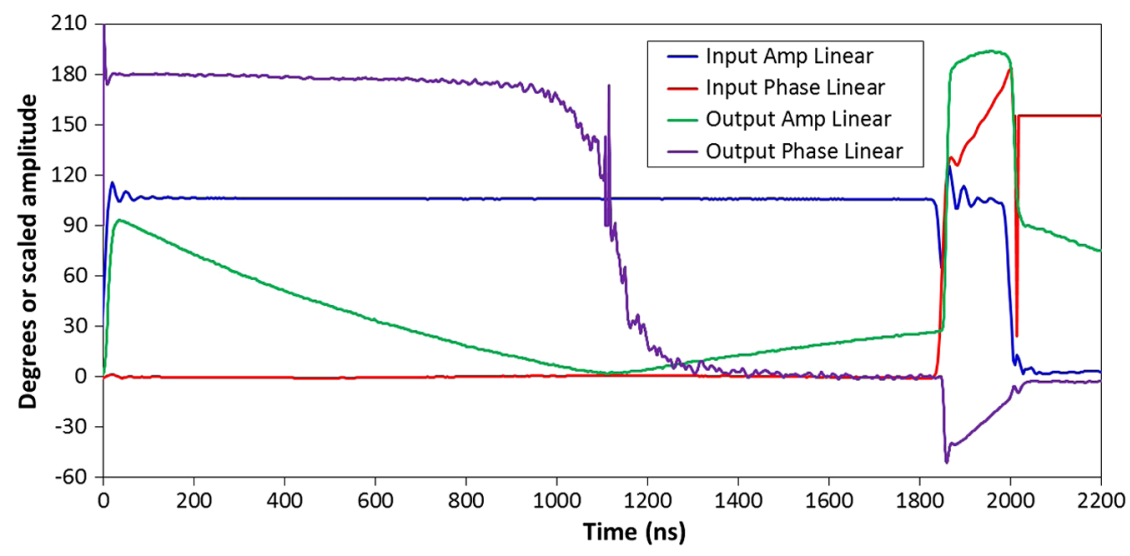

FIG. 14. Measured input and output to and from the pulse compressor at XBOX-3 when a phase switch of $120^{\circ}$, followed by a linear phase ramp is applied to the drive signal to a $7 \mathrm{MW}$ klystron. 


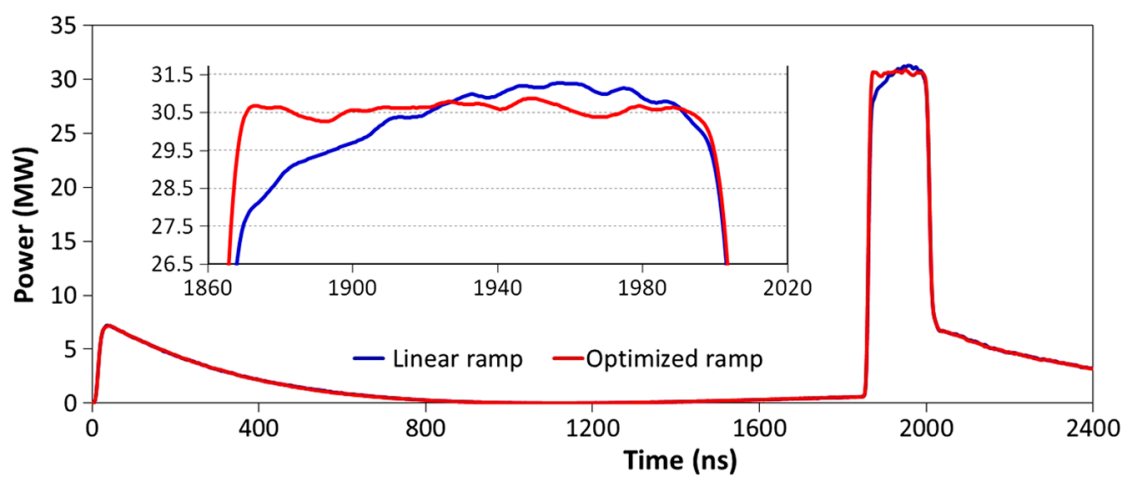

FIG. 15. Comparison of measured output pulse shape at XBOX-3 for a linear phase ramp and an optimized phase; inset shows compressed pulse top.

smaller but significant phase transient. The output phase is difficult to measure near to $1120 \mathrm{~ns}$ as the amplitude goes to zero and hence the plot has significant noise at this point. For the linear phase ramp it can be seen that the output pulse does not have the desired flattop for high gradient testing. The power is determined as the square of the amplitude therefore the power variation is larger as a fraction than the amplitude variation.

Figure 15 looks at the performance of the optimizing controller using the algorithm of Eq. (25). The set point used in Eq. (25) has taken the average power of the compressed pulse for the linear ramp. The starting linear ramp gave an output with a $3 \mathrm{MW}$ variation in $30 \mathrm{MW}$. The optimized ramp takes a few tens of pulses to evolve and has a flattop variation of about $0.6 \mathrm{MW}$ in $30 \mathrm{MW}$.

For an ideal pulse compressor and transmission system where there are no unwanted reflections, a smooth phase ramp on the input will give a smooth amplitude output. Furthermore, a flat amplitude output can be achieved with an input phase ramp following a low order polynomial. Feedback control is applied to the input on the supposition that the output should be smooth. For the control performance presented in Fig. 15 derivatives of the output amplitude during the compressed pulse have been determined by fitting a fifth order polynomial through points occurring $1.25 \mathrm{~ns}$ or more after the amplitude has reached $90 \%$ of its peak value; this peak value is as determined by a $\sim 10 \mathrm{~ns}$ rolling average.

This phase program gives a peak amplitude just after the center of the compressed pulse. Before the controller is applied, the phase jump is adjusted to get the required average amplitude. If the peak amplitude must not exceed a set value then initial adjustment can use the peak value.

The compressed pulse power output for the linear ramp curve shown in Fig. 15 has undulations on a time scale of 15 ns along what should be a smooth, curved peak. Part of the unevenness comes from sampling errors. The biggest contribution comes from the klystron transient seen in Fig. 14 as the curve labeled "input amp linear." One might have hoped that the controller could compensate for the klystron transient. The nonlinear nature of the klystron response makes perfect compensation very difficult to find. The optimized ramp in Fig. 15 has a variation at the level of $1 \%$ from the desired value. This performance is adequate for high gradient testing. Were this pulse to be fed to a traveling wave accelerator cavity then it is the average power over the transit time for a disturbance to propagate through the structure at its group velocity that matters. This averaging process would reduce gradient variations below input power variations. A smoother flattop can be achieved at XBOX-2 as transients on the input after phase switching are smaller. Transients on the E37113 klystron can be reduced by reducing the rate of phase change demanded on the phase ramp.

\section{CONCLUSION}

Improvements in the design and control of CERN's high power $12 \mathrm{GHz}$ test stands has enabled better control of the conditioning procedure for high gradient structures. As acceleration structures are easily damaged during conditioning it is important that the procedure can be automated for repeatability when structures are to be produced on an industrial scale. This paper has established a theoretical framework for feed forward controllers applied to SLED-1 pulse compressors. The work has demonstrated by simulation and measurement that the control algorithms proposed work effectively and robustly.

The demonstrated use of X-band pulse compressors to generate accurately controlled, high output powers from multiple low power klystrons makes affordable, high gradient, X-band linacs a feasible option for future user facilities.

\section{ACKNOWLEDGMENTS}

This work has been supported by STFC ST/G008248/1 and the European Union 7th Framework Programme, Grant No. 227579 EuCARD. This work has been made possible by the CERN technical staff and colleagues who have developed and established the XBOX test stands. 
[1] A. Grudiev, S. Calatroni, and W. Wuensch, New local field quantity describing the high gradient limit of accelerating structures, Phys. Rev. ST Accel. Beams 12, 102001 (2009).

[2] A. Grudiev, D. Schulte, and W. Wuensch, Report No. CERN-AB-2006-028, 2006.

[3] N. Catalan-Lasheras et al., Report No. CERN-ACC-20140166, 2014.

[4] A. M. Sesslerand S. S. Yu, Relativistic Klystron Two-Beam Accelerator, Phys. Rev. Lett. 58, 2439 (1987).

[5] A. Vlieks, Report No. SLAC-PUB-13741, 2009.

[6] D. Farkas, H. A. Hogg, G. A. Loew, and P. B. Wilson, SLED: A method for doubling SLAC's energy, In Proceedings of 9th International Conference on High Energy Accelerators, SLAC (1974), p. 576.

[7] P. B. Wilson, Z. D. Farkas, and R. D. Ruth, SLEDII: A new method of rf pulse compression, LINAC, Albuquerque, New Mexico, 1990, http://accelconf.web.cern.ch/ accelconf/190/papers/mo456.pdf.

[8] C. Nantista, Doctoral dissertation, UCLA, 1994, http://www .slac.stanford.edu/pubs/slacreports/reports02/slac-r-455.pdf.

[9] S. G. Tantawi, C. Adolphsen, S. Holmes, T. Lavine, R. J. Loewen, C. Nantista, C. Pearson, R. Pope, J. Rifkin, R. D. Ruth and, A.E. Vlieks, The next linear collider test accelerator's rf pulse compression and transmission systems, in Proceedings of the Particle Accelerator Conference, Vancouver, BC, Canada, 1997 (IEEE, New York, 1997).

[10] T. Shintake and N. Akasaka, A new rf pulse compressor using multicell coupled cavity system (No. KEK-96-71), SCAN-9609133.

[11] T. Sugimura, T. Kamitani, K. Yokoyama, K. Kakihara, M. Ikeda, and S. Ohsawa, SKIP-A pulse compressor for SUPERKEKB, in Proceedings of LINAC 2004, Lübeck, Germany, http://accelconf.web.cern.ch/Accelconf/104/ PAPERS/THP61.PDF.

[12] S. G. Tantawi, C. D. Nantista, V. A. Dolgashev, C. Pearson, J. Nelson, K. Jobe, J. Chan, K. Fant, J. Frisch, and D. Atkinson, High-power multimode X-band rf pulse compression system for future linear colliders, Phys. Rev. ST Accel. Beams 8, 042002 (2005).

[13] R. Bossart, P. Brown, J. Mourier, I. V. Syratchev, and L. Tanner, CLIC Note 592, Report No. CERN-OPEN-2004015, http://cds.cern.ch/record/741401/files/open-2004-015 .pdf.

[14] P. Brown and I. Syratchev, $3 \mathrm{GHz}$ barrel open cavity (BOC) rf pulse compressor for CTF3, Microwave Symposium Digest, 2004 IEEE MTT-S International (IEEE, New York, 2004), Vol. 2.
[15] P. Wang, I. Syratchev, H. Zha, W. Wuensch, and H. Chen, The design of a compact, high power pulse compressor with a flat output pulse, IPAC2016, Busan, Korea, THPMW022, http://accelconf.web.cern.ch/accelconf/ ipac2016/papers/thpmw022.pdf.

[16] H. Zha and A. Grudiev, Design and optimization of compact linear collider main linac accelerating structure, Phys. Rev. Accel. Beams 19, 111003 (2016).

[17] S. Döbert, Report No. SLAC-PUB-10690, 2004, http://www .slac.stanford.edu/cgi-wrap/getdoc/slac-pub-10690.pdf.

[18] V. A. Dolgashev, Progress on high-gradient structures, AIP Conf. Proc. 1507, 76 (2012).

[19] C. Nantista, Z. D. Farkas, N. M. Kroll, T. L. Lavine, A. Menegat, R. D. Ruth, S. G. Tantawi, A. E. Vlieks, and P. B. Wilson, High power rf pulse compression with SLED-II at SLAC, in Proceedings of the 15th Particle Accelerator Conference, PAC-1993, Washington, DC, 1993 (IEEE, New York, 1993).

[20] B. J. Woolley, Ph.D. thesis, Lancaster University, 2015

[21] S. Y. Kazakov, X-band TE10-TE01 mode converters, in Proceedings of the 17th International Linear Accelerator Conference (LINAC-1994), Tsukuba, Japan, 1994 (KEK, Tsukuba, Japan, 1994).

[22] C. Nantista, Overmoded waveguide components for high-power rf, in Proceedings of the 6th workshop on high energy density and high power rf, Berkley Springs, WV, 2003, http://www.slac.stanford.edu/cgi-wrap/getdoc/ slac-pub-10218.ps.gz.

[23] A. Fiebig and C. Schieblich, CERN Report Nos. CERNPS-90-13-RF, CM-P00059378, 1990, https://cds.cern.ch/ record/209756/files/CM-P00059378.pdf.

[24] S. H. Shaker, R. Corsini, P. K. Skowronski, I. Syratchev, and F. Tecker, Phase modulation programming to get flat pulses with desired length and power from the CRF3 pulse compressors, in Proceedings of the International Particle Accelerator Conference, Kyoto, Japan (ICR, Kyoto, 2010), TUPEA043.

[25] C. Serpico, M. Dal Forno, and A. Fabris, Optimization of the SLED phase modulation parameters of the Fermi Linac, in Proceedings of the 25th Particle Accelerator Conference, PAC-2013, Pasadena, CA, 2013 (IEEE, New York, 2013), WEPMA01.

[26] G. Shu, F.-L. Zhao, S.-L. Pei, and O.-Z. Xiao, rf modulation studies on an $\mathrm{S}$ band pulse compressor, Chin. Phys. 40, 037002 (2016).

[27] A. C. Dexter, Time domain SLED-1 pulse compressor controller simulation code, Lancaster University, 2017, http://dx.doi.org/10.17635/Lancaster/researchdata/163. 